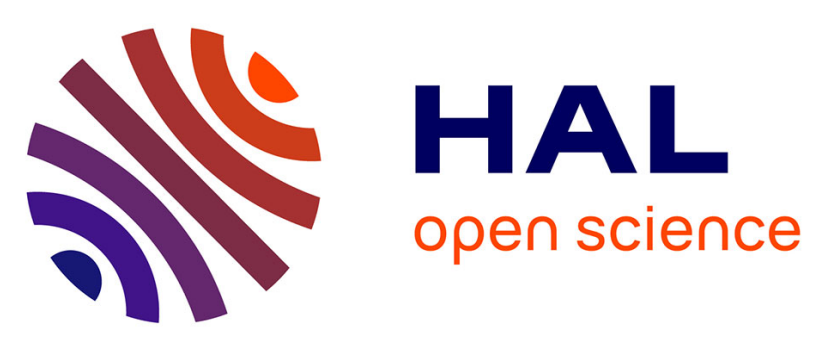

\title{
ULTRASONIC SPECTROSCOPY IN CROSS-PLY FIBER REINFORCED COMPOSITES APPLIED TO DISPERSION EFFECTS CHARACTERIZATION OF ELASTIC SHEAR WAVE
}

T. Lhermitte, Bernard Perrin, M. Fink

\section{To cite this version:}

T. Lhermitte, Bernard Perrin, M. Fink. ULTRASONIC SPECTROSCOPY IN CROSS-PLY FIBER REINFORCED COMPOSITES APPLIED TO DISPERSION EFFECTS CHARACTERIZATION OF ELASTIC SHEAR WAVE. Journal de Physique Colloques, 1990, 51 (C2), pp.C2-1265-C2-1268. 10.1051/jphyscol:19902297 . jpa-00230638

\section{HAL Id: jpa-00230638 https://hal.science/jpa-00230638}

Submitted on 1 Jan 1990

HAL is a multi-disciplinary open access archive for the deposit and dissemination of scientific research documents, whether they are published or not. The documents may come from teaching and research institutions in France or abroad, or from public or private research centers.
L'archive ouverte pluridisciplinaire $\mathbf{H A L}$, est destinée au dépôt et à la diffusion de documents scientifiques de niveau recherche, publiés ou non, émanant des établissements d'enseignement et de recherche français ou étrangers, des laboratoires publics ou privés. 
ler Congrès Français d'Acoustique 1990

\title{
ULTRASONIC SPECTROSCOPY IN CROSS-PLY FIBER REINFORCED COMPOSITES APPLIED TO DISPERSION EFFECTS CHARACTERIZATION OF ELASTIC SHEAR WAVE
}

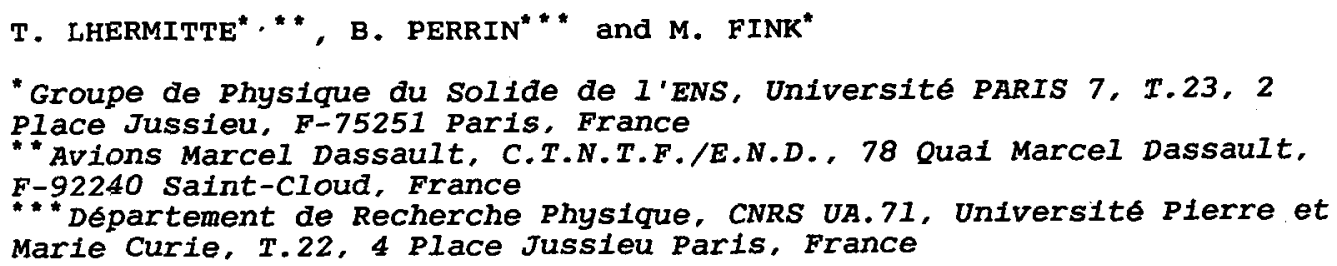

Résumé - Une analyse théorique et expérimentale des effets de dispersion, lors de la propagation d'ondes élastiques transversales, est présentée dans deux composites carbone/résine à plis croisés. Ceux-ci possèdent respectivement, dans la limite des grandes longueurs d'ondes, un axe de rotation d'ordre 4 et un axe de rotation d'ordre 8 . Lorsque la longueur d'onde élastique devient comparable à la période du composite, des effets de dispersion spatiaux apparaîssent : déviation de linéarité des courbes de dispersion, bandes d'arrêt et, pour le matériau possèdañt un axe de rotation d'ordre 8 , une activité acoustique induite par l'empilement helicoidal des couches élémentaires.

\begin{abstract}
The theoretical and experimental studies of the dispersion effects, for the propagation of elastic shear waves, in two cross-ply fiber reinforced composites are presented. They exhibit, in the long wavelength limit, $a$ fourfold and an eightfold axis of rotation, respectively. When the elastic wavelength becomes comparable to the composite period, spatial dispersion effects are expected : departure from the linearity of the curves, stop bands and, in the eigthfold axis material, an acoustical activity induced by the helical stacking of the elementary layers.
\end{abstract}

\section{1 - Introduction}

The cross-ply fiber reinforced composites which we have studied are made of two phases : an organic matrix (epoxy resin) and carbon reinforcement fibers with high mechanical characteristics. These materials are manufactured by stacking elementary layers in predetermined directions with a given sequence which corresponds to a superperiod. The elementary layer, with a thickness $L=150 \mu \mathrm{m}$, is made of a random distribution of pre-impregnated fibers, oriented along a single axis, which forms the basic building block of the material.

Two cross-ply composites, labeled I and II, consisting of a large number of plies, have been investigated. The superperiod of the composite I is made of two orthogonal elementary layers (Fig.1). The composite II, represented on Fig.2, is obtained by stacking four layers with a $\pi / 4$ helicoidal step. After polymerization of the resin, two laminated and periodic materials, with respective periods $2 L$ and $4 L$, are obtained. They have, in the long wavelength limit $(\lambda>>L)$, a fourfold and an eigthfold axis of rotation respectively, which are both normal to the fibers direction.

In this work, we report a theoretical study and an experimental analysis through an ultrasonic spectroscopy method, of the dispersion effects for elastic shear waves propagating along the symmetry axis of these two cross-ply composites. Spatial dispersion effects are expected when the wavelentgh becomes comparable to the thickness of the superperiod, and appear for frequencies as low as a few $\mathrm{MHz}$.

In the composite I, the shear wave propagating along the fourfold axis is doubly degenerated. As usual, the spatial dispersion appears through the existence of forbidden frequencies or stop bands in the dispersion curve ${ }^{1}$.

In the composite $I$, the shear waves are no longer degenerated. The helicity of the stacking involves the existence of two waves with respective right and left polarizations and different velocities. A new effect, which can be described by acoustical activity ${ }^{2}$ in the long wavelength limit, is therefore added to the above 
pattern and appears through the existence of semi-gaps in the dispersion curve. In these bands, one of the two polarizations is propagative while the other one is forbidden.

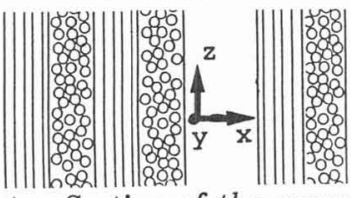

Fig.1 : Section of the composite

I. The layers are stacked 0/90.

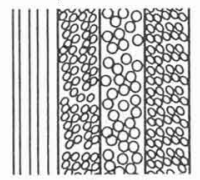

Fig.2 : Section of the composite II. The layers are stacked 0/45/90/-45.

\section{2 - Theoretical part}

The dispersion relations, for the propagation of a plane shear elastic wave with frequency $\omega$ along the $x$ axis of the stacking (Fig.1 and Fig.2), can be derived from the eigenvalues of the transfer-matrices $T_{I}$ and $T_{I I}$ for a superperiod of the composites I and II respectively.

These two matrices have two eigenvalues respectively, either complexe conjugate of unit modulus, or real with one the inverse of the other. The complexe solutions define respectively a forward and backward propagative wave for frequencies corresponding to a propagative shear wave of wavevector $k$ through the composite. The real solution of modulus greater than 1 is eliminated for physical reasons while the other one of modulus lower than 1 defines an evanescent wave for frequencies which correspond to stop bands.

\section{Composite I}

As expected, an elastic degenerated shear wave can propagate in this composite. From the complex conjugate eigenvalues $\exp ( \pm 2 i k L)$ of $T_{I}$, we have obtained the well known dispersion relation ${ }^{3}$ which remains also valid for longitudinal waves :

$$
\begin{gathered}
\cos (k \mathcal{L})=\left(1+\eta^{2}\right) \cos (\omega \mathcal{L} / s)-\eta^{2} \cos \left(\eta \omega \mathcal{L} / s^{\prime}\right) \\
\text { with }\left\{\begin{array} { c } 
{ s ^ { \prime } = \sqrt { s _ { \| / } s _ { \perp } } } \\
{ s = ( 2 s _ { / / } s _ { \perp } ) / ( s _ { \| / } + s _ { \perp } ) }
\end{array} \text { and } \left\{\begin{array}{c}
\eta=\left(s_{/ /}-s_{\perp}\right) / 2 s^{\prime} \\
\mathcal{L}=2 L
\end{array}\right.\right.
\end{gathered}
$$

$s_{/ /}$and $s_{\perp}$ represent the velocities, corresponding to polarization directions parallel and normal respectively to the fibers direction direction, of a shear wave propagating through the thickness of a ply.

The dispersion curve is represented on Fig.3. The gaps corresponding to the condition $|\cos (k \mathcal{L})|>1$ appear at the edge of each Brillouin zone for $k=p \pi / \mathcal{L}, p=1,2, \ldots$

\section{Composite II}

In this case, there is no more degeneracy. Due to the helicoidal stacking of the layers, two waves with right and left polarizations, and no rectilinear one, exist in the medium.

The dispersion relation is then obtained from the complex conjugate eigenvalues : $\exp ( \pm i(4 k L+(2 q+1) \pi / 4))$ of the matrix $T_{I I}$ as :

$$
\begin{gathered}
\cos (k L+(2 q+1) \pi / 4)=\frac{1}{2 \sqrt{2}}\left[\left(\cos \left(k_{/ /} L\right)+\cos \left(k_{\perp} L\right)\right) \pm \delta\right] \quad(2) \text { where } \\
\delta=\left[\left(\cos \left(k_{/ /} L\right)+\cos \left(k_{\perp} L\right)\right)^{2}-6 \cos \left(k_{/ /} L\right) \cos \left(k_{\perp} L\right)+\frac{r^{2}+1}{r} \sin \left(k_{/ /} L\right) \sin \left(k_{\perp} L\right)+2\right]^{1 / 2}
\end{gathered}
$$

with $k_{\gamma}=\omega / s_{\gamma}, \gamma=1, / /, r=k_{\perp} / k_{/ /}, \mathrm{q}:$ an integer whose value depends on the frequency band

considered.

Fig.4 represents the dispersion curve in the reduced Brillouin zone. This curve displays two waves propagating in the medium with an acoustical activity which appears when the wavelength becomes comparable to the superperiod and increases with frequency. The two waves have respectively left and right circular polarizations at low frequencies. At higher frequencies; a right and left direction can always be attributed to each of them, except in a few points of degeneracy. 


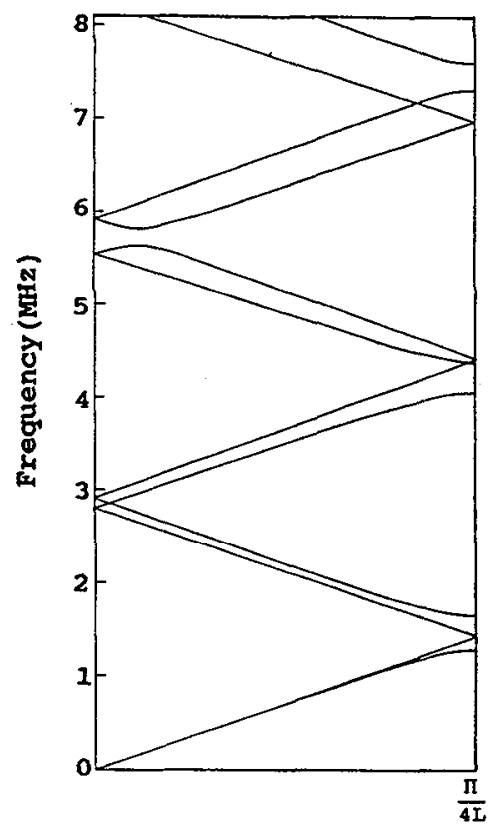

Wavenumber $\mathbf{k}$

Fig.4 : Theoretical dispersion curve of the composite II for elastic shear wave propagating normal to the stacking.

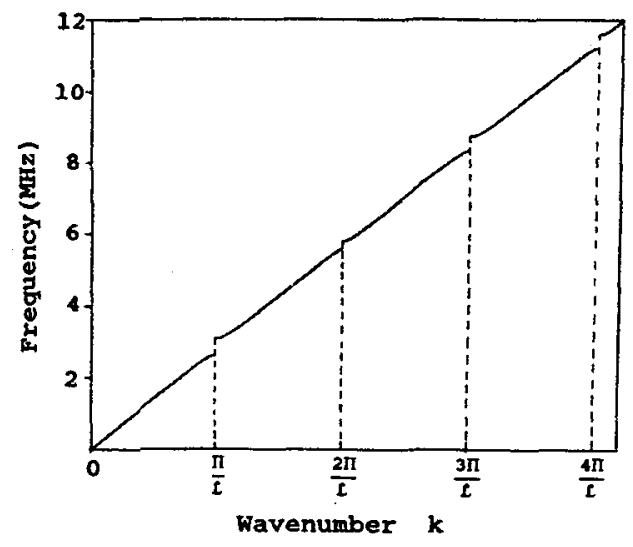

Fig.3 : Theoretical dispersion curve of the composite I for elastic shear wave propagating normal to the stacking.

In the frequency domain $[1.26 \mathrm{MHz}<\nu<1.64 \mathrm{MHz}]$, a semi-gap of type $|\cos (k L+\pi / 4)|>1$ appears. In this band, the two waves $(+\delta)$ and $(-\delta)$ in Eq.2 are forbidden and propagative respectively. At higher frequency, the two polarizations are propagative until the band [ $4.02 \mathrm{MHz}<\nu<4.32 \mathrm{MHz}$ ], where a semigap of type $|\cos (k L+\pi / 4)|>1$ appears again. In this one, the forbidden wave of the previous semi-gap becomes propagative while the other one is now forbidden. Finally for $[5.52 \mathrm{MHz}<\nu<5.91 \mathrm{MHz}]$, we have a full gap which corresponds to the condition $\delta^{2}<0$.

\section{3 - Experimental part}

The fiber composites under investigation are of type T300/ 914, carbon-reinforced epoxy, of density $1577 \mathrm{~kg} / \mathrm{m}^{3}$ and mass fraction of resin $35 \%$, built by Avions Marcel Dassault Company.

Two samples have been manufactured : one composite of type $I$ in which each ply unit is stacked $0 / 90$ to form a quadratic material made of 50 superperiods (100 plies i.e. $15 \mathrm{~mm}$ thick) and one composite of type II constituted of 16 superperiods (64 plies i.e. $9.6 \mathrm{~mm}$ thick). In the first one, the stacking is $0 / 45 / 90 /-45$. In the second one, a mirror in the stacking sequence of the elementary layers has been introduced. This mirror corresponds to an inversion of the helicity of the stacking which leads to the following sequences : $0 / 45 / 90 /-45$ for the eight first superperiods and $-45 / 90 / 45 / 0$ for the eight last ones. The introduction of this mirror, as it will be seen in the discussion, is a trick way to display the semi-gaps relative to the two polarizations on the same spectrum.

In order to show the existence of gaps in the frequency domain, a spectroscopy method using broadband pulses was chosen. In our experimental set-up, shear wave transducers and ultrasonic transmission technique were used. Two pairs of shear wave broadband transducers, with respective nominal centerfrequencies of $20 \mathrm{MHz}$ and $5 \mathrm{MHz}$, were successively applied to the cross-ply composites and axially compressed to a constant load. The two transducers (transmitter and receiver) are fixed on both sides of the sheets and their polarization directions oriented parallel. A broadband pulse is used to excite the transmitter. The received signal is then amplified, filtered, numerized and averaged by a digital oscilloscope and its spectrum computed using a FFT algorithm.

The spectra of the received temporal signals are not full, as those we can obtain in unidirectional composites for longitudinal waves for instance. They possess gaps for frequencies corresponding to the stop bands 
shown in the theoretical part.

For the composite I, the experimental evidence for the existence of gaps is shown in Fig.5a and Fig.5b

For the composite II, the transducer receives a temporal signal relative to the two waves. Therefore its spectrum does not allow us to display the gaps for these two polarizations. A mirror have then been introduced as indicated above. This mirror kills the propagative polarization, which is alternatively either right or left, in order to restore only the gaps, represented on Fig.6a and Fig.6b, corresponding to the forbidden polarization.

The spectra obtained with the first pair of transducers of center-frequency of $5 \mathrm{MHz}$ take a little into account the bandwidth frequency effect. For the second pair of center-frequency of $20 \mathrm{MHz}$, the bandwidth effect is more important and involves then a little deformation of the depth of the gaps. Therefore, a qualitative experimental approach of the gaps is given. Moreover, the ultrasonic attenuation, quiet important in these materials reduces their width and their depth.
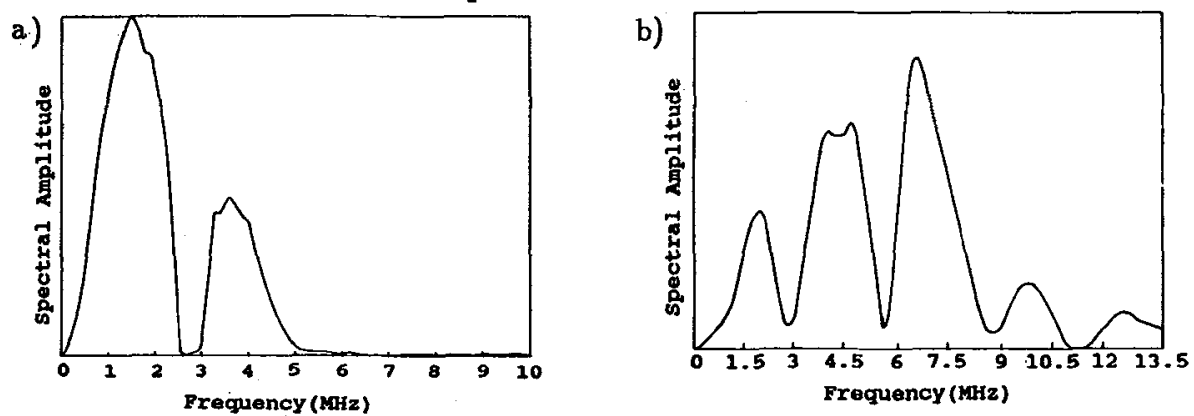

Fig.5 : Spectrum of a transmission pulse for the composite $I$, in arbitrairy unit. The polarizations of the transducers are parallel. a).The transducers have a center- frequency of $5 \mathrm{MHz}$. The first gap is put in evidence. b).The transducers have a center-frequency of $20 \mathrm{MHz}$. The four first gaps are put in evidence.
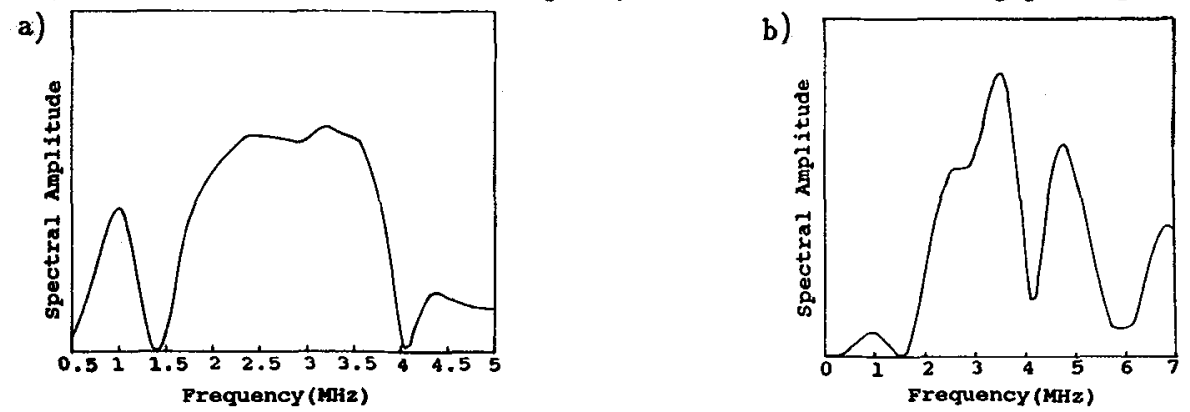

Fig.6 : Spectrum of a transmission pulse, in arbitrairy unit, for the composite II in which a mirror is introduced. The polarizations of the transducers are parallel. a).The transducers have a center-frequency of $5 \mathrm{MHz}$. The two first semi-gaps are put in evidence. b).The transducers have a center-frequency of 20MHz. The two first semi-gaps and the first full gap are put in evidence.

\section{4 - Conclusion}

First theoretical and experimental results on the dispersion effects, for the propagation of elastic shear waves in 0/90 and 0/45/90/-45 fiber-reinforced composites are reported. These effects, which appear when the wavelength becomes comparable to the superperiod, do not proceed from a mass difference of the elementary layers, but are only due to their specific stacking.

Acknowledgements : The authors gratefully acknowledge the support of Avions Marcel Dassault Company, especially R. De Mol and C. Soufflet for helpfull comments and friendly advice.

References

${ }^{3}$ L. Brillouin, "Wave Propagation in Periodic Structures", Dover Publications, Inc., 1953.

${ }^{2}$ D.L. Portigal and E. Burstein, "Acoustical activity and other first-order spatial dispersion effects in crystals", J. Phys. Rev. 170, 673-678, 1968.

${ }^{3}$ L.M. Brekhovskikh, "Waves in layered media", Academic Press, New-York, 1960. 